TRANSACTIONS OF THE

AMERICAN MATHEMATICAL SOCIETY

Volume 361, Number 2, February 2009, Pages 793-818

S 0002-9947(08)04711-9

Article electronically published on September 29, 2008

\title{
THE ABSOLUTELY CONTINUOUS SPECTRUM OF DISCRETE CANONICAL SYSTEMS
}

\author{
ANDREAS FISCHER AND CHRISTIAN REMLING
}

\begin{abstract}
We prove that if the canonical system $J\left(y_{n+1}-y_{n}\right)=z H_{n} y_{n}$ has absolutely continuous spectrum of a certain multiplicity, then there is a corresponding number of linearly independent solutions $y$ which are bounded in a weak sense.
\end{abstract}

\section{INTRODUCTION}

In this paper, we want to study the absolutely continuous spectrum of higher order difference equations from a general point of view. We consider so-called canonical systems:

$$
J\left(y_{n+1}-y_{n}\right)=z H_{n} y_{n} .
$$

Here, $J, H_{n} \in \mathbb{C}^{2 d \times 2 d}, y_{n} \in \mathbb{C}^{2 d}, z \in \mathbb{C}, H_{n} \geq 0$ (as a quadratic form on $\mathbb{C}^{2 d}$ ) and $J=\left(\begin{array}{cc}0 & -1 \\ 1 & 0\end{array}\right)$. We further assume that $H_{n} J H_{n}=0$. This latter assumption is essential to make sure that the boundary value problems associated with (1.1) are formally symmetric.

Canonical systems provide a very general framework; for example, we will later show that every formally symmetric scalar equation of arbitrary (even) order can be put in this form.

Here is one form of our main result. The precise definition of the set $S_{m} \subset \mathbb{R}$ will be given later.

Theorem 1.1. Let $S_{m}$ be the set on which (1.1) has absolutely continuous spectrum of (exact) multiplicity $m(1 \leq m \leq d)$. Let $n_{j} \in \mathbb{N}$ be an arbitrary sequence with $\lim _{j \rightarrow \infty} n_{j}=\infty$. Then for almost all $\lambda \in S_{m}$, there are $d+m$ linearly independent solutions $y_{1}, \ldots, y_{d+m}$ of $J\left(y_{k}(n+1)-y_{k}(n)\right)=\lambda H(n) y_{k}(n)$ with

$$
\liminf _{j \rightarrow \infty} y_{k}^{*}\left(n_{j}\right) H\left(n_{j}\right) y_{k}\left(n_{j}\right)<\infty
$$

for $k=1, \ldots, d+m$.

Loosely speaking, this means that there are $d+m$ solutions that are bounded (albeit in a weak sense) if there is absolutely continuous spectrum of multiplicity $m$. This neatly confirms general (and rather vague) notions about the absolutely continuous spectrum. Namely, corresponding to absolutely continuous spectrum

Received by the editors March 7, 2007.

2000 Mathematics Subject Classification. Primary 39A70, 34B05, 34L05.

Key words and phrases. Canonical systems, absolutely continuous spectrum.

The second author's work was supported by the Heisenberg program of the Deutsche Forschungsgemeinschaft. 
of multiplicity $m$, there should be $2 m$ solutions of roughly constant size, $d-m$ decaying and $d-m$ growing solutions. This indeed leads to $2 m+d-m=d+m$ solutions whose size does not increase, as asserted by Theorem 1.1 .

Theorem 1.1 is a generalization of a fundamental result of Last and Simon [10, Theorem 1.2]. Last and Simon deal with second order scalar equations, corresponding to $d=1$ in (1.1). To prove Theorem 1.1, we will follow the strategy of [10]. The fact that the spectrum need no longer be simple leads to new issues that have to be addressed. In particular, we will need to study in detail the effects of a variation of the boundary conditions at the left endpoint. This may be reformulated as a problem in complex symplectic linear algebra. This problem is solved in Sect. 6 .

We have chosen the framework of canonical systems because we want to be as general as possible since Theorem 1.1 deals with structural aspects of an absolutely continuous spectrum. At least in the second order case, (continuous) canonical systems have indeed proven to be fundamental from several points of view. For example, de Branges spaces are always generated by canonical systems [4, 15]. Moreover, the customary equations (Sturm-Liouville, Dirac, Jacobi) may all be written as canonical systems. For more on the theory of (continuous) canonical systems of arbitrary order $2 d$, we refer the reader to [1, 16]. The literature on the second order case $(d=1)$ is considerably larger. We just mention 8 and refer the reader to the references quoted therein.

We do not know of any systematic treatment of discrete canonical systems, so we develop the material we need from scratch. The main difficulty is that it is not at all obvious how to define self-adjoint operators whose eigenvalue equations are given by (1.1). We will take the treatment of [15, Sect. 10] as a guideline.

It is even more difficult to clarify the relations between the different possible definitions of the spectral measures of half line problems. We do not attempt a deep analysis of this question in this paper. For us, the main point is to ensure that the absolutely continuous part of this spectral measure remains invariant under a change of boundary conditions. Thus, we adopt an armchair approach and use a definition that makes a result of Gesztesy and Tsekanovskii [7] applicable, which will give us the desired invariance.

The issues mentioned in the preceding three paragraphs will be discussed in Sect. 2-5. Sect. 6 is central to our treatment; here, we study questions from complex symplectic linear algebra. We can then prove Theorem 1.1 in Sect. 7. Finally, in Sect. 8, we try to further justify our choice of canonical systems as the general framework by showing that any scalar equation of even order $2 d$ can be written in the form (1.1).

\section{Spectral TheORY ON Finite INTERVALS}

In this section, we want to study eigenvalue problems associated with equation (1.1) on a finite interval $n \in\{1, \ldots, N\}$. We work with the (finite-dimensional) Hilbert space $\ell_{2}^{H}(\{1, \ldots, N\})$. Its elements are equivalence classes of functions $y:\{1, \ldots, N\} \rightarrow \mathbb{C}^{2 d}$, the scalar product is given by $\langle y, z\rangle=\sum_{n=1}^{N} y_{n}^{*} H_{n} z_{n}$, and functions $y, y^{\prime}$ with $\left\|y-y^{\prime}\right\|=0$ are identified in $\ell_{2}^{H}$.

There are several obvious problems. First of all, the operator associated with (1.1) should formally be given by $(T y)_{n}=H_{n}^{-1} J\left(y_{n+1}-y_{n}\right)$, but $H_{n}$ is not invertible. If one tries to define $T y=f$ by requiring the difference equation $J\left(y_{n+1}-y_{n}\right)=H_{n} f_{n}$ to hold, then it is neither clear that any $y \in \ell_{2}^{H}$ has an 
image $f=T y$ under $T$ nor is it clear that this image (if it exists) is well defined, since different representatives of $y \in \ell_{2}^{H}$ might lead to different images $f$.

These issues will be addressed in this section. We will basically follow the method of [15, Sect. 10].

We will first define boundary value problems associated with (1.1) by hand and only later link things up with operators on (subspaces of) the Hilbert space $\ell_{2}^{H}$.

We start out by establishing a formula of the same type as Green's identity. Such a formula is essential if one wants to describe the self-adjoint realizations in terms of boundary conditions. Suppose that the difference equations

$$
J\left(y_{n+1}-y_{n}\right)=H_{n} f_{n}, \quad J\left(z_{n+1}-z_{n}\right)=H_{n} g_{n}
$$

are satisfied for $n=1, \ldots, N$. Then

$$
\langle y, g\rangle_{\ell_{2}^{H}(\{1, \ldots, N\})}-\langle f, z\rangle_{\ell_{2}^{H}(\{1, \ldots, N\})}=\left.y_{n}^{*} J z_{n}\right|_{n=1} ^{n=N+1} .
$$

If we interpret $f$ as $T y$ and $g$ as $T z$, then (2.1) expresses $\langle y, T z\rangle-\langle T y, z\rangle$ as a difference of two symplectic forms that involve the values of $y, z$ at the boundaries only.

Equation (2.1) is proved by the following calculation:

$$
\begin{aligned}
\langle y, g\rangle-\langle f, z\rangle & =\sum_{n=1}^{N} y_{n}^{*} H_{n} g_{n}-\sum_{n=1}^{N} f_{n}^{*} H_{n} z_{n} \\
& =\sum_{n=1}^{N} y_{n}^{*} H_{n} g_{n}-\sum_{n=1}^{N} f_{n}^{*} H_{n}\left(z_{n+1}+J H_{n} g_{n}\right) \\
& =\sum_{n=1}^{N} y_{n}^{*} H_{n} g_{n}-\sum_{n=1}^{N} f_{n}^{*} H_{n} z_{n+1} \\
& =\sum_{n=1}^{N} y_{n}^{*} J\left(z_{n+1}-z_{n}\right)+\sum_{n=1}^{N}\left(y_{n+1}^{*}-y_{n}^{*}\right) J z_{n+1} \\
& =-\sum_{n=1}^{N} y_{n}^{*} J z_{n}+\sum_{n=1}^{N} y_{n+1}^{*} J z_{n+1} \\
& =y_{N+1}^{*} J z_{N+1}-y_{1}^{*} J z_{1} .
\end{aligned}
$$

To pass to the third line, we have used the fact that $H_{n} J H_{n}=0$.

We now want the boundary forms $y^{*} J z$ to vanish separately at $n=1$ and $n=N+1$ ("separated boundary conditions"). We seek maximal subspaces with this property. More specifically, we want to work with subspaces $L \subset \mathbb{C}^{2 d}$ with $v^{*} J w=0$ for all $v, w \in L$, and $L$ should be maximal with this property. These so-called Lagrangian subspaces admit the following description: Let $\alpha_{1}, \alpha_{2} \in \mathbb{C}^{d \times d}$ with

$$
\alpha_{1} \alpha_{1}^{*}+\alpha_{2} \alpha_{2}^{*}=1, \quad \alpha_{1} \alpha_{2}^{*}-\alpha_{2} \alpha_{1}^{*}=0 .
$$

Then $L_{\alpha}:=\left\{v \in \mathbb{C}^{2 d}:\left(\alpha_{1}, \alpha_{2}\right) v=0\right\}$ is a Lagrangian subspace, $L_{\alpha} \neq L_{\beta}$ if $\alpha \neq \beta$ both satisfy (2.2), and every Lagrangian subspace arises in this way. We will prove this characterization of Lagrangian subspaces in Sect. 6, where we will also discuss other questions from symplectic linear algebra. See also [3, Chapter 11] or [2. 
We can now try to associate boundary value problems with the canonical system (1.1). The following definition suggests itself: Fix $\alpha, \beta$ satisfying (2.2) and impose the boundary conditions

$$
\left(\alpha_{1}, \alpha_{2}\right) y_{1}=0, \quad\left(\beta_{1}, \beta_{2}\right) y_{N+1}=0 .
$$

We call $z \in \mathbb{C}$ an eigenvalue of (1.1), (2.3) if there is a non-trivial solution $y$ to these equations. One can of course just work with this definition, but we will try to get additional insight by identifying the set of these eigenvalues as the spectrum of a self-adjoint operator in a subspace of $\ell_{2}^{H}$.

There are only finitely many eigenvalues, so we can certainly fix a number $z_{0} \in \mathbb{C}$ that is not an eigenvalue. We will consider the resolvent at the point $z_{0}$ (which is formally given by $\left(T-z_{0}\right)^{-1}$ ) and construct the sought operator $T$ from this object. To simplify the notation, we suppose that $z_{0}=0$; this amounts to assuming that the matrix $\left(\begin{array}{ll}\alpha_{1} & \alpha_{2} \\ \beta_{1} & \beta_{2}\end{array}\right)$ is regular. Then, if $\left(f_{j}\right)_{j=1}^{N}$ is given, there is a unique solution $y$ of the inhomogenous equation $J\left(y_{n+1}-y_{n}\right)=f_{n}(n=1, \ldots, N)$ that satisfies the boundary conditions (2.3). A straighforward calculation shows that this solution can be obtained with the help of a kernel $K$ as

$$
y_{n}=\sum_{j=1}^{N} K(n, j) f_{j},
$$

where

$$
K(n, j)=\left\{\begin{array}{ll}
B_{\alpha \beta} J-J, & j<n, \\
B_{\alpha \beta} J, & j \geq n,
\end{array} \quad B_{\alpha \beta}=\left(\begin{array}{ll}
\alpha_{1} & \alpha_{2} \\
\beta_{1} & \beta_{2}
\end{array}\right)^{-1}\left(\begin{array}{cc}
0 & 0 \\
\beta_{1} & \beta_{2}
\end{array}\right) .\right.
$$

From the construction of $K$, it is clear that $z$ is an eigenvalue with a corresponding eigenfunction $y$ precisely if

$$
y_{n}=z \sum_{j=1}^{N} K(n, j) H_{j} y_{j} .
$$

This equation also makes sense in the Hilbert space $\ell_{2}^{H}(\{1, \ldots, N\})$ because the right-hand side only depends on the equivalence class $\widetilde{y}$ of $y$ (recall that $y \sim 0$ $\left.\Longleftrightarrow H_{n} y_{n} \equiv 0\right)$. More precisely, the kernel $K$ defines an operator $\mathcal{K} H$ on $\ell_{2}^{H}$ by (2.4). If $y$ is an eigenvector with eigenvalue $z$, then $\widetilde{y}=z \mathcal{K} H \widetilde{y}$. Conversely, if this equation in $\ell_{2}^{H}$ holds, then there is a unique representative $y$ (namely, the one defined by (2.4) ) which is an eigenfunction corresponding to the eigenvalue $z$. In particular, it follows that different eigenfunctions also represent different elements of $\ell_{2}^{H}$

Define $\ell_{2}^{I}$ similarly to $\ell_{2}^{H}$, but with $H$ replaced by the identity matrix $I \in \mathbb{C}^{2 d \times 2 d}$ (so $\ell_{2}^{I}$ is isomorphic to a $2 d$-fold orthogonal sum of $\ell_{2}$ spaces of scalar valued functions). Let $V$ be the isometry

$$
V: \ell_{2}^{H} \rightarrow \ell_{2}^{I}, \quad(V y)_{n}=H_{n}^{1 / 2} y_{n}
$$

Here, $H_{n}^{1 / 2}$ is the non-negative square root of $H_{n} \geq 0$. Let $\mathcal{L}: \ell_{2}^{I} \rightarrow \ell_{2}^{I}$ be the operator with kernel

$$
L(n, j)=H_{n}^{1 / 2} K(n, j) H_{j}^{1 / 2} .
$$

Lemma 2.1. $L(n, j)=L(j, n)^{*}$ and $\mathcal{L}$ is self-adjoint. 
Proof. The second claim follows immediately from the identity for the kernel. This identity, in turn, follows from the corresponding identity for $K$,

$$
K(n, j)-K(j, n)^{*}=J \delta_{n j},
$$

and the fact that $H_{n} J H_{n}=0$. So we need only prove (2.5).

Let $y, z$ satisfy the boundary conditions (2.3) and solve the equations

$$
J\left(y_{n+1}-y_{n}\right)=f_{n}, \quad J\left(z_{n+1}-z_{n}\right)=g_{n} .
$$

Then

$$
\begin{aligned}
0 & =\sum_{n=1}^{N}\left(y_{n+1}^{*} J z_{n+1}-y_{n}^{*} J z_{n}\right)=\sum_{n=1}^{N}\left(\left(y_{n}^{*}+f_{n}^{*} J\right) J\left(z_{n}-J g_{n}\right)-y_{n}^{*} J z_{n}\right) \\
& =\sum_{n=1}^{N}\left(f_{n}^{*} J g_{n}+y_{n}^{*} g_{n}-f_{n}^{*} z_{n}\right)=\sum_{j, n=1}^{N} f_{n}^{*}\left[J \delta_{n j}-K(n, j)+K(j, n)^{*}\right] g_{j} .
\end{aligned}
$$

As $f, g$ are arbitrary, (2.5) follows.

We observed above that the eigenvalues and eigenfunctions are precisely the solutions of (2.4). Also, it is possible to view (2.4) as an equation in the Hilbert space $\ell_{2}^{H}$. In the following lemma, we will further reformulate this condition.

Lemma 2.2. Let $f \in \ell_{2}^{I}, z \neq 0$. The following statements are equivalent:

a) $\mathcal{L} f=z^{-1} f$

b) $f \in R(V)$ and the unique $y \in \ell_{2}^{H}$ with $f=V y$ satisfies $y=z \mathcal{K} H y$.

Here, $R(V) \subset \ell_{2}^{I}$ denotes the range of $V$.

Proof. We see from the form of the kernel $L$ that $R(\mathcal{L}) \subset R(V)$. Now if a) holds, then $f=z \mathcal{L} f$ lies in $R(V)$; hence $f=V y$ for $y \in \ell_{2}^{H}$. So $f_{n}=H_{n}^{1 / 2} y_{n}$ and $z(\mathcal{L} f)_{n}=z H_{n}^{1 / 2} \sum_{j=1}^{N} K(n, j) H_{j} y_{j}$ and thus

$$
H_{n}^{1 / 2}\left(y_{n}-z \sum_{j=1}^{N} K(n, j) H_{j} y_{j}\right)=0 .
$$

In other words, $y=z \mathcal{K} H y$ in $\ell_{2}^{H}$.

Conversely, if b) holds, then we get a) by multiplying from the left by $H_{n}^{1 / 2}$ on both sides of $y=z \mathcal{K} H y$.

Let $P$ be the orthogonal projection onto $R(V)$ in $\ell_{2}^{I}$. Note that

$$
R(V)^{\perp}=\left\{f \in \ell_{2}^{I}: \sum_{n=1}^{N} g_{n}^{*} H_{n}^{1 / 2} f_{n}=0 \quad \forall g \in \ell_{2}^{I}\right\}=\left\{f \in \ell_{2}^{I}:\|f\|_{\ell_{2}^{H}}=0\right\} .
$$

Thus $\mathcal{L}(1-P)=0$. On the other hand, since $R(P)=R(V) \supset R(\mathcal{L})$, we have that $P \mathcal{L}=\mathcal{L}$. It follows that $P \mathcal{L}=\mathcal{L} P$, and thus $R(V)$ is a reducing subspace for the self-adjoint operator $\mathcal{L}$. Let $\mathcal{L}_{0}$ be the restriction of $\mathcal{L}$ to $R(V)$. Then, since $\mathcal{L}(1-P)=0$ (as noted above), $\mathcal{L}=\mathcal{L}_{0} \oplus 0$.

The crucial step in the construction of a self-adjoint operator $T$ associated with (1.1), (2.3) is the introduction of the following subspace. Define

$$
Z=\left\{f \in \ell_{2}^{H}: \exists y \text { with }\|y\|_{\ell_{2}^{H}}=0, J\left(y_{n+1}-y_{n}\right)=H_{n} f_{n}, y \text { satisfies (2.3) }\right\} .
$$


Since such sequences $y$ represent the zero element of $\ell_{2}^{H}$, we can interpret $Z$ as the space of the images of zero of the sought "operator" (more precisely, of a relation). In the following lemma, we write $N\left(\mathcal{L}_{0}\right)$ for the kernel of $\mathcal{L}_{0}$.

Lemma 2.3. $N\left(\mathcal{L}_{0}\right)=V Z$.

Proof. Let $g \in N\left(\mathcal{L}_{0}\right)$. Recalling that $\mathcal{L}_{0}$ acts in $R(V)$, we can write $g=V f$ with $f \in \ell_{2}^{H}$. Then $H_{n}^{1 / 2} \sum_{j=1}^{N} K(n, j) H_{j} f_{j}=0$. Put $y_{n}=\sum_{j=1}^{N} K(n, j) H_{j} f_{j}$. Then, as just observed, $H_{n} y_{n}=0$, and by the construction of $K, y$ satisfies the boundary conditions and the equation $J\left(y_{n+1}-y_{n}\right)=H_{n} f_{n}$. Hence $f \in Z$ and $g \in V Z$.

Conversely, if $f \in Z$ and $g=V f$, then there exists a sequence $y$ that satisfies the boundary conditions, the equation $J\left(y_{n+1}-y_{n}\right)=H_{n} f_{n}$, and $H_{n} y_{n}=0$. We again use the properties of $K$ to represent $y$ as $y_{n}=\sum_{j=1}^{N} K(n, j) H_{j} f_{j}$. Hence $\mathcal{L}_{0} g=\mathcal{L}_{0} V f=H_{n}^{1 / 2} y_{n}=0$.

Theorem 2.4. The (normalized) eigenfunctions of

$$
J\left(y_{n+1}-y_{n}\right)=z H_{n} y_{n}, \quad\left(\alpha_{1}, \alpha_{2}\right) y_{1}=\left(\beta_{1}, \beta_{2}\right) y_{N+1}=0
$$

form an orthonormal basis of the Hilbert space $\ell_{2}^{H} \ominus Z$.

Proof. Obviously, the (normalized) eigenfunctions of the self-adjoint operator $\mathcal{L}_{0}$ corresponding to non-zero eigenvalues form an orthonormal basis of $R(V) \ominus N\left(\mathcal{L}_{0}\right)$. Apply the unitary map $V^{-1}: R(V) \rightarrow \ell_{2}^{H}$ and use Lemmas 2.2, 2.3.

We have succeeded in relating the eigenvalue problem (1.1), (2.3) to the spectral theory of a self-adjoint operator in a Hilbert space. We now show that a more direct line of attack leads to the same result. Define the relation $\mathcal{R}_{0}$ by

$$
\mathcal{R}_{0}=\left\{(y, f): J\left(y_{n+1}-y_{n}\right)=H_{n} f_{n}, y \text { satisfies the boundary conditions }\right\} .
$$

Equivalently, $(y, f) \in \mathcal{R}_{0}$ if and only if

$$
y_{n}=\sum_{j=1}^{N} K(n, j) H_{j} f_{j} .
$$

Also define

$$
\mathcal{R}=\left\{(\widetilde{y}, \widetilde{f}):(y, f) \in \mathcal{R}_{0}\right\} \subset \ell_{2}^{H} \oplus \ell_{2}^{H} .
$$

(We use a tilde if we want to emphasize that we are considering elements of $\ell_{2}^{H}$.) Our goal is to extract an operator from the relation $\mathcal{R}$. Now for a given $y \in \ell_{2}^{H}$, it might happen that $(y, f) \notin \mathcal{R}$ for all $f \in \ell_{2}^{H}$, and even if $(y, f) \in \mathcal{R}$ for suitable $f \in \ell_{2}^{H}$, this $f$ might not be unique. We are thus led to introducing the spaces

$$
D=\left\{y \in \ell_{2}^{H}: \exists f \in \ell_{2}^{H} \text { with }(y, f) \in \mathcal{R}\right\}
$$

(the projection of $\mathcal{R}$ onto the first component) and, as above,

$$
Z=\left\{f \in \ell_{2}^{H}:(0, f) \in \mathcal{R}\right\} .
$$

We now claim that $(\widetilde{y}, \widetilde{f}) \in \mathcal{R} \Longleftrightarrow V \widetilde{y}=\mathcal{L}_{0} V \widetilde{f}$. Indeed, if this latter relation holds, then $\widetilde{y}=\mathcal{K} H \tilde{f}$. Take an arbitrary representative $f \in \tilde{f}$ and define a representative $y$ of $\widetilde{y}$ by (2.6). Then $(y, f) \in \mathcal{R}_{0}$ and thus $(\widetilde{y}, \widetilde{f}) \in \mathcal{R}$. The converse is proved by reversing these steps. 
Recall that $V$ maps $\ell_{2}^{H}$ unitarily onto $R(V) \subset \ell_{2}^{I}$. So $V$ is invertible as a map to $R(V)$, and thus

$$
(y, f) \in \mathcal{R} \Longleftrightarrow y=V^{-1} \mathcal{L}_{0} V f .
$$

In particular, it follows that $Z=N\left(V^{-1} \mathcal{L}_{0} V\right)$, and, since this operator is selfadjoint,

$$
D=R\left(V^{-1} \mathcal{L}_{0} V\right)=N\left(V^{-1} \mathcal{L}_{0} V\right)^{\perp}=Z^{\perp}=\ell_{2}^{H} \ominus Z .
$$

We obtain the self-adjoint operator

$$
T: D \rightarrow D, \quad T=\left(\left.\left(V^{-1} \mathcal{L}_{0} V\right)\right|_{D}\right)^{-1}
$$

from the relation $\mathcal{R}_{0}$, just as in Theorem 2.4. The eigenvalues and eigenfunctions of the boundary value problem from Theorem 2.4 are exactly the eigenvalues and eigenvectors of $T$.

A vector $\widetilde{y} \in \ell_{2}^{H}$ lies in $D$ if and only if there exists a representative $y \in \widetilde{y}$ and an $f$, so that $y$ satisfies the boundary conditions and $J\left(y_{n+1}-y_{n}\right)=H_{n} f_{n}$. In this case, $y$ is unique and the unique $\widetilde{f}$ with the properties from above and, in addition, $\widetilde{f} \in D$, is the image $\widetilde{f}=T \widetilde{y}$.

\section{Spectral measures}

As the underlying Hilbert space $\mathcal{H}=D=\ell_{2}^{H} \ominus Z$ is finite-dimensional, the operator $T$ introduced in the preceding section has a purely discrete spectrum, and a spectral representation can thus be obtained by expanding in terms of eigenfunctions. We proceed as follows. Let $u(n, z)$ be the solution of (1.1) with the initial value $u(1, z)=\left(\begin{array}{c}-\alpha_{2}^{*} \\ \alpha_{1}^{*}\end{array}\right)$ (we write $u(n)$ instead of $u_{n}$ here, and we will continue to change between these two notation). So $u$ satisfies the boundary condition at $n=1$, and the columns of $u$ span the space of solutions of (1.1) with this additional property. We further introduce the map $U$ by

$$
(U f)(\lambda)=\sum_{n=1}^{N} u^{*}(n, \lambda) H(n) f(n) .
$$

In other words, $U$ computes the scalar products with the solutions $u(\cdot, \lambda)$. The goal is to introduce a spectral measure $\rho$ that makes $U$ unitary onto $L_{2}(\mathbb{R}, d \rho)$. If $\lambda$ is an eigenvalue, we can find a matrix $P_{\lambda} \in \mathbb{C}^{d \times d}$ so that the columns of $u(\cdot, \lambda) P_{\lambda}$ span the eigenspace $N(T-\lambda)$. We can actually assume that $P_{\lambda}$ is an orthogonal projection. Put $N_{\lambda}=\sum_{n=1}^{N} u^{*}(n, \lambda) H(n) u(n, \lambda)$. The compression of $N_{\lambda}$ to $R\left(P_{\lambda}\right), P_{\lambda} N_{\lambda} P_{\lambda}$, is invertible as an operator on $R\left(P_{\lambda}\right)$. Indeed, $v^{*} N_{\lambda} v>0$ for all $v \in R\left(P_{\lambda}\right)$ because eigenfunctions cannot have zero norm. Define

$$
\rho=\sum P_{\lambda}\left(P_{\lambda} N_{\lambda} P_{\lambda}\right)^{-1} P_{\lambda} \delta_{\lambda} .
$$

The sum is over the eigenvalues, the inverse really means the inverse in $R\left(P_{\lambda}\right)$, as just explained, and $\delta_{\lambda}$ is the Dirac measure at $\lambda$.

Theorem 3.1. $U: \mathcal{H} \rightarrow L_{2}(\mathbb{R}, d \rho)$ is unitary.

Note that $\rho$ is a matrix valued measure. The scalar product in $L_{2}(\mathbb{R}, d \rho)$ is given by $\langle f, g\rangle=\int f^{*}(\lambda) d \rho(\lambda) g(\lambda)$ (in this order). 
Proof. Let $E$ be an eigenvalue and consider first the case $f(n)=u(n, E) a$ with $a \in R\left(P_{E}\right)$. Then the following evaluation holds almost everywhere with respect to $\rho:$

$$
\begin{aligned}
(U f)(\lambda) & =\sum_{n=1}^{N} u^{*}(n, \lambda) H(n) u(n, E) a=\sum_{n=1}^{N} P_{\lambda} u^{*}(n, \lambda) H(n) u(n, E) a \\
& =\delta_{\lambda E} P_{E} N_{E} P_{E} a .
\end{aligned}
$$

For the last equality, we use the fact that eigenfunctions corresponding to different eigenvalues are orthogonal and that $P u^{*}=(u P)^{*}$ and the columns of $u P$ are eigenfunctions.

Hence, if $g(n)=u\left(n, E^{\prime}\right) b$ with an eigenvalue $E^{\prime}$ and $b \in R\left(P_{E^{\prime}}\right)$, then

$$
\begin{aligned}
\langle U f, U g\rangle_{L_{2}(\mathbb{R}, d \rho)} & =\delta_{E E^{\prime}} a^{*} P_{E} N_{E} P_{E}\left(P_{E} N_{E} P_{E}\right)^{-1} P_{E} N_{E} P_{E} b=\delta_{E E^{\prime}} a^{*} N_{E} b \\
& =\delta_{E E^{\prime}} a^{*} \sum_{n=1}^{N} u^{*}(n, E) H(n) u(n, E) b=\langle f, g\rangle_{\ell_{2}^{H}}=\langle f, g\rangle_{\mathcal{H}} .
\end{aligned}
$$

Since the functions $f=u(\cdot, E) a$ span $\mathcal{H}, U$ is isometric. Since the images $U f=$ $\delta_{\lambda E} P_{E} N_{E} P_{E} a$ span $L_{2}(\mathbb{R}, d \rho), U$ is unitary.

We can also consider the $U$ defined in (3.1) as an operator from $\ell_{2}^{H}$ to $L_{2}(\mathbb{R}, d \rho)$. Then $U$ is a partial isometry. More precisely, the following holds.

Theorem 3.2. $N(U)=Z$.

Proof. Since we know already that $U$ is unitary from $\mathcal{H}=Z^{\perp}$ to $L_{2}(\mathbb{R}, d \rho)$, we must show that $U f=0$ for all $f \in Z$. So let $f \in Z$. By the definition of $Z$, there exists a sequence $y$ so that $J\left(y_{n+1}-y_{n}\right)=H_{n} f_{n}, H_{n} y_{n}=0(n=1, \ldots, N)$, and $y$ satisfies the boundary conditions. Now Green's identity (2.1) shows that

$$
(U f)(\lambda)=\sum_{n=1}^{N} u_{n}^{*}(\lambda) H_{n} f_{n}=\lambda \sum_{n=1}^{N} u_{n}^{*} H_{n} y_{n}+\left.u_{n}^{*} J y_{n}\right|_{n=1} ^{n=N+1}=u_{N+1}^{*} J y_{N+1} .
$$

We have used the fact that $u_{1}^{*} J y_{1}=0$, since $u$ and $y$ both satisfy the boundary condition at $n=1$.

We are interested in $(U f)(\lambda)=u^{*}(N+1, \lambda) J y(N+1)$ as an element of $L_{2}(\mathbb{R}, d \rho)$, so we may restrict $\lambda$ to the eigenvalues. Moreover, the projections $P_{\lambda}$ from (3.2) ensure that $(U f)(\lambda)=\left(u(N+1, \lambda) P_{\lambda}\right)^{*} J y(N+1)$ almost everywhere with respect to $\rho$. In other words, only eigenfunctions (and not arbitrary linear combinations of the columns of $u$ ) need to be considered. But these eigenfunctions satisfy the boundary conditions at $n=N+1$, as does $y$, hence $\left(u(N+1, \lambda) P_{\lambda}\right)^{*} J y(N+1)=0$.

The following observation is an immediate consequence of the fact that $U$ is a partial isometry. It will be a crucial input to the method of Last-Simon [10].

Corollary 3.3. For all $f \in \ell_{2}^{H}(\{1, \ldots, N\})$, we have that $\|U f\|_{L_{2}(\mathbb{R}, d \rho)} \leq\|f\|_{\ell_{2}^{H}}$.

Actually, we will use another version of this:

Theorem 3.4. Let $P_{n}$ be the orthogonal projection onto $R\left(H_{n}\right) \subset \mathbb{C}^{2 d}$. Then for $n=1, \ldots, N$,

$$
\int_{\mathbb{R}} H_{n}^{1 / 2} u(n, \lambda) d \rho(\lambda) u^{*}(n, \lambda) H_{n}^{1 / 2} \leq P_{n}
$$


Proof. Fix an arbitrary $w \in \mathbb{C}^{2 d}$ and put $f_{k}=w \delta_{k n}$. Then $(U f)(\lambda)=u^{*}(n, \lambda) H_{n} w$, and thus Corollary 3.3 shows that

$$
w^{*} \int_{\mathbb{R}} H_{n} u(n, \lambda) d \rho(\lambda) u^{*}(n, \lambda) H_{n} w \leq w^{*} H_{n} w
$$

or, equivalently,

$$
\left(H_{n}^{1 / 2} w\right)^{*} \int_{\mathbb{R}} H_{n}^{1 / 2} u(n, \lambda) d \rho(\lambda) u^{*}(n, \lambda) H_{n}^{1 / 2} H_{n}^{1 / 2} w \leq\left(H_{n}^{1 / 2} w\right)^{*} P_{n} H_{n}^{1 / 2} w .
$$

So, if we denote the matrix from the left-hand side of (3.3) by $I$, then (3.4) says that $v^{*} I v \leq v^{*} P_{n} v$ for all $v \in R\left(H_{n}\right)$. For $v \in R\left(H_{n}\right)^{\perp}=N\left(H_{n}\right)$, obviously $I v=P_{n} v=0$, so the proof is complete.

\section{4. $M$ FUnCTIONS}

In this section, we present an alternate approach to constructing the spectral measure $\rho$. Namely, we introduce and use Titchmarsh-Weyl $M$ functions. For this theory in various different situations, see [2, 3, 9, 14].

In this approach, one does not introduce operators, but works directly with equation (1.1). Let $Y(\cdot, z)$ be a fundamental matrix of (1.1), with the initial value $Y(1, z)=\left(\begin{array}{cc}\alpha_{1}^{*} & -\alpha_{2}^{*} \\ \alpha_{2}^{*} & \alpha_{1}^{*}\end{array}\right)$. So the last $d$ columns of the $2 d \times 2 d$ matrix $Y$ are just the solution $u$ introduced above. Write $Y=(v, u)$ and put, for $M \in \mathbb{C}^{d \times d}$,

$$
F_{M}(n, z)=Y(n, z)\left(\begin{array}{c}
1 \\
M
\end{array}\right)=v(n, z)+u(n, z) M
$$

As usual, the Titchmarsh-Weyl $M$ function of the problem (1.1), (2.3) is defined by requiring that $F_{M}$ satisfy the boundary condition at $n=N+1$. More precisely, for $z \in \mathbb{C}^{+}=\{z \in \mathbb{C}: \operatorname{Im} z>0\}$, we demand that $\left(\beta_{1}, \beta_{2}\right) F_{M}(N+1, z)=0$. This defines a matrix $M(z)=M_{\alpha, \beta}^{(N)}(z)$. Note that such an $M$ must exist because otherwise there would be non-real eigenvalues. Also, $M$ is unique. In fact, writing $v=\left(\begin{array}{l}v_{1} \\ v_{2}\end{array}\right)$ and $u=\left(\begin{array}{l}u_{1} \\ u_{2}\end{array}\right)$, we can express $M$ as

$$
M(z)=-\left(\beta_{1} u_{1}(N+1, z)+\beta_{2} u_{2}(N+1, z)\right)^{-1}\left(\beta_{1} v_{1}(N+1, z)+\beta_{2} v_{2}(N+1, z)\right) .
$$

This representation shows that $M$ is holomorphic on $\mathbb{C}^{+}$; in fact, it is a rational function.

For $z \in \mathbb{C}^{+}$and $M \in \mathbb{C}^{d \times d}$ (not necessarily equal to one of the matrices $M_{\alpha, \beta}^{(N)}(z)$ from above), introduce

$$
E_{z}(M)=\operatorname{Im} z \sum_{n=1}^{N} F_{M}^{*}(n, z) H(n) F_{M}(n, z)-\operatorname{Im} M,
$$

with $\operatorname{Im} M=(1 / 2 i)\left(M-M^{*}\right)$.

Theorem 4.1. Let $z \in \mathbb{C}^{+}, M \in \mathbb{C}^{d \times d}$. Then $M=M_{\alpha, \beta}^{(N)}(z)$ for some boundary condition $\beta$ if and only $E_{z}(M)=0$.

Proof. We claim that

$$
E_{z}(M)=\frac{1}{2 i} F_{M}^{*}(N+1, z) J F_{M}(N+1, z) .
$$


This follows from Green's identity (2.1). Indeed, with $y=z=F_{M}(\cdot, z)$ and thus $f=g=z F_{M}(\cdot, z)$, we see that

$$
\begin{aligned}
F_{M}^{*}(N+1, z) J F_{M}(N+1, z) & \\
& =F_{M}^{*}(1, z) J F_{M}(1, z)+2 i \operatorname{Im} z \sum_{n=1}^{N} F_{M}^{*}(n, z) H(n) F_{M}(n, z) .
\end{aligned}
$$

Moreover,

$$
\begin{aligned}
F_{M}^{*}(1, z) J F_{M}(1, z) & =\left(1, M^{*}\right)\left(\begin{array}{cc}
\alpha_{1} & \alpha_{2} \\
-\alpha_{2} & \alpha_{1}
\end{array}\right) J\left(\begin{array}{cc}
\alpha_{1}^{*} & -\alpha_{2}^{*} \\
\alpha_{2}^{*} & \alpha_{1}^{*}
\end{array}\right)\left(\begin{array}{c}
1 \\
M
\end{array}\right) \\
& =M^{*}-M=-2 i \operatorname{Im} M,
\end{aligned}
$$

and hence (4.2) holds.

Now if $M=M_{\beta}(z)$ for some boundary condition $\beta$, then, by the construction of $M_{\beta}$, the solution $F_{M_{\beta}}$ satisfies the boundary condition $\beta$ at $n=N+1$ and hence $E_{z}\left(M_{\beta}\right)=0$ by (4.2) (because the boundary conditions single out those subspaces on which the form $u^{*} J v$ vanishes).

Conversely, if $E_{z}(M)=0$, set

$$
\left(\gamma_{1}, \gamma_{2}\right)=\left(1, M^{*}\right) Y^{*}(N+1, z) J
$$

with $\gamma_{1,2} \in \mathbb{C}^{d \times d}$. Then $\left(\gamma_{1}, \gamma_{2}\right) F_{M}(N+1, z)=0$. Moreover,

$$
\gamma_{1} \gamma_{2}^{*}-\gamma_{2} \gamma_{1}^{*}=-\left(\gamma_{1}, \gamma_{2}\right) J\left(\begin{array}{c}
\gamma_{1}^{*} \\
\gamma_{2}^{*}
\end{array}\right)=-F_{M}^{*}(N+1, z) J F_{M}(N+1, z)=0
$$

by (4.2). Since $\left(\gamma_{1}, \gamma_{2}\right)$ has full rank (equal to $d$ ), $A:=\gamma_{1} \gamma_{1}^{*}+\gamma_{2} \gamma_{2}^{*}$ is an invertible matrix. Put $\beta_{j}=A^{-1 / 2} \gamma_{j}(j=1,2)$. The above observations imply that $\left(\beta_{1}, \beta_{2}\right)$ is an admissible boundary condition (this is to say, (2.2) holds) and

$$
\left(\beta_{1}, \beta_{2}\right) F_{M}(N+1, z)=0 .
$$

In other words, $M=M_{\beta}(z)$.

For second order equations, the sets

$$
\mathcal{C}_{\alpha}^{(N)}(z)=\left\{M_{\beta}(z): \beta \text { boundary condition }\right\}=\left\{M \in \mathbb{C}^{d \times d}: E_{z}(M)=0\right\}
$$

are circles in the complex plane $(d=1$ here). If $N$ increases, these circles are nested. Here, we have more complicated objects, but they are still nested in the following sense: Introduce the sets ("discs")

$$
\mathcal{D}_{\alpha}^{(N)}(z)=\left\{M \in \mathbb{C}^{d \times d}: E_{z}(M) \leq 0\right\} .
$$

Then $\mathcal{D}_{\alpha}^{\left(N_{1}\right)}(z) \supset \mathcal{D}_{\alpha}^{\left(N_{2}\right)}(z)$ if $N_{1} \leq N_{2}$. This follows at once from (4.1).

Equation (4.1) also shows that $M_{\beta}$ is a Herglotz function. This means that $M_{\beta}(z)$ is defined and holomorphic on the upper half plane and $\operatorname{Im} M_{\beta}(z) \geq 0$ there (in the sense that the matrix is positive definite). Consequently, there exist matrices $A, B \in \mathbb{C}^{d \times d}, A=A^{*}, B \geq 0$, and a (matrix valued) positive Borel measure $\nu$ on $\mathbb{R}$ with $\int_{\mathbb{R}} d(\operatorname{trace} \nu)(t) /\left(t^{2}+1\right)<\infty$, so that

$$
M_{\beta}(z)=A+B z+\int_{\mathbb{R}}\left(\frac{1}{t-z}-\frac{t}{t^{2}+1}\right) d \nu(t) .
$$


$A, B$ and $\nu$ are uniquely determined by $M_{\beta}$. Occasionally, it is useful to have finite measures. To this end, one can also write

$$
M_{\beta}(z)=A+\int_{\overline{\mathbb{R}}} \frac{1+t z}{t-z} d \mu(t),
$$

with $\overline{\mathbb{R}}=\mathbb{R} \cup\{\infty\}$ and

$$
d \mu(t)=\frac{d \nu(t)}{t^{2}+1}+B \delta_{\infty} .
$$

The representation (4.4) has the additional advantage that $\mu$ is a measure on the compact space $\overline{\mathbb{R}}$.

We now relate the $M$ functions discussed above to the material from Sect. 3.

Theorem 4.2. The measure $\nu$ from the Herglotz representation (4.3) of $M_{\beta}$ is the spectral measure $\rho$ from (3.2).

This does not come as a suprise; on the contrary, this fact is one of the main points of the Weyl construction.

Proof. We compute the norm of $F=F_{M_{\beta}}$ in two ways. First of all, by (4.1) and Theorem 4.1,

$$
\sum_{n=1}^{N} F(n, z)^{*} H(n) F(n, z)=\frac{\operatorname{Im} M_{\beta}(z)}{\operatorname{Im} z} .
$$

Next, we use the partial isometry $U$ from (3.1). We use a convenient matrix notation: $U F$ is the matrix whose columns are given by $U$ applied to the corresponding columns of $F$. Green's identity (2.1) shows that in $L_{2}(\mathbb{R}, d \rho)$,

$$
\begin{aligned}
(\lambda-z)(U F)(\lambda) & =(\lambda-z) \sum_{n=1}^{N} u^{*}(n, \lambda) H(n) F(n, z)=-\left.u^{*}(n, \lambda) J F(n, z)\right|_{n=1} ^{N+1} \\
& =u^{*}(1, \lambda) J F(1, \lambda)=\left(-\alpha_{2}, \alpha_{1}\right) J\left(\begin{array}{c}
\alpha_{1}^{*}-\alpha_{2}^{*} M_{\beta}(z) \\
\alpha_{2}^{*}+\alpha_{1}^{*} M_{\beta}(z)
\end{array}\right)=1
\end{aligned}
$$

The boundary term at $n=N+1$ vanishes because of the argument at the end of the proof of Theorem 3.2. Almost everywhere with respect to $\rho$, we only need to consider eigenvalues $\lambda$ and eigenfunctions $\left(u(\cdot, \lambda) P_{\lambda}\right)^{*}$, and these eigenfunctions as well as $F$ satisfy the boundary condition at $n=N+1$.

Now the material from Sect. 3 shows that

$$
\sum_{n=1}^{N} F(n, z)^{*} H(n) F(n, z)=\int_{\mathbb{R}} \frac{d \rho(\lambda)}{|\lambda-z|^{2}}+\sum_{n=1}^{N}\left(P_{Z} F\right)^{*}(n, z) H(n)\left(P_{Z} F\right)(n, z),
$$

where $P_{Z}$ denotes the projection onto $Z \subset \ell_{2}^{H}$. To analyze $P_{Z} F$, fix a sequence $f \in Z$. By definition of $Z$, there exists $y$ so that $J\left(y_{n+1}-y_{n}\right)=H_{n} f_{n}, H_{n} y_{n}=0$, and $y$ satisfies the boundary conditions. A calculation similar to the one used in the proof of Theorem 3.2 shows that

$$
\sum_{n=1}^{N} F^{*}(n, z) H(n) f(n)=-F^{*}(1, z) J y(1) .
$$


By plugging in the value of $F(1, z)$, we may further evaluate this as

$$
\sum_{n=1}^{N} F^{*}(n, z) H(n) f(n)=\left(-\alpha_{2}, \alpha_{1}\right) y(1) .
$$

In particular, the scalar product of (a column of) $F(\cdot, z)$ with any vector from $Z$ is independent of $z \in \mathbb{C}^{+}$. Consequently, $P_{Z} F(\cdot, z)$ is also independent of $z$. By combining this result with (4.5), (4.6), we obtain

$$
\operatorname{Im} M_{\beta}(z)=B \operatorname{Im} z+\operatorname{Im} z \int_{\mathbb{R}} \frac{d \rho(t)}{|t-z|^{2}},
$$

where $B \geq 0$ is a constant matrix. But the measure from the Herglotz representation is uniquely determined by $\operatorname{Im} M$, hence comparison with (4.3) shows that $\nu=\rho$; in fact (referring to (4.4) ), we have that $d \mu(t)=d \rho(t) /\left(t^{2}+1\right)+B \delta_{\infty}$.

\section{Spectral measures For halF Line Problems}

We are now given an equation of the form (1.1) on a half line $n \in \mathbb{N}$, together with a boundary condition $\alpha$ at $n=1$, and we want to introduce spectral measures for this problem. This seems to be a rather subtle problem, as there are several reasonable looking possible definitions, and the relations between them are not at all clear. For example, one might be satisfied with measures $\rho$ for which the map $U$ becomes a partial isometry from $\ell_{2}^{H}(\{1, \ldots, N\})$ to $L_{2}(\mathbb{R}, d \rho)$ for all $N \in \mathbb{N}$. Or one might require these maps to be isometric on the spaces $\ell_{2}^{H}(\{1, \ldots, N\}) \ominus Z_{N}$. In this case, one would probably only consider situations in which these spaces are subspaces of one another. One could also try to construct self-adjoint operators corresponding to the half line problem and then consider the spectral measures of these operators.

Here, we will take a rather pedestrian approach modelled on the construction of spectral measures in the classical Weyl theory. More precisely, we will consider limit points of spectral measures of problems on $\{1, \ldots, N\}$ for $N \rightarrow \infty$. The following observation will get us started. It will be convenient to work with the measures $\mu=\mu_{\beta}^{(N)}$ from (4.4) instead of $\rho$.

Lemma 5.1. There is a constant $C$ so that trace $\mu_{\beta}^{(N)}(\overline{\mathbb{R}}) \leq C$ for all $N \in \mathbb{N}$ and all boundary conditions $\beta$.

Proof. Equation (4.4) shows that $\mu_{\beta}^{(N)}(\overline{\mathbb{R}})=\operatorname{Im} M_{\beta}^{(N)}(i)$, and these matrices are uniformly bounded because by the nesting property, all $M_{\beta}^{(N)}(i)$ lie in the compact set $\mathcal{D}^{(1)}(i)$.

Now the Banach-Alaoglu Theorem ensures that there are weak $*$ convergent subsequences $\mu_{\beta_{j}}^{\left(N_{j}\right)} \rightarrow \mu$. We transform back and dismiss a possible discrete point at infinity and call every measure $\rho$ on (the Borel sets of) $\mathbb{R}$ of the form $d \rho(t)=$ $\left(t^{2}+1\right) d \mu(t)$, with such a weak $*$ limit point $\mu$, a spectral measure of the half line problem. Lemma 5.1 shows that spectral measures always exist.

This definition is rather general, and we cannot expect $\rho$ to have many properties. It is true, however, that $U$ from (3.1) maps $\ell_{2}^{H}(\mathbb{N})$ contractively into (but, in general, certainly not onto) $L_{2}(\mathbb{R}, d \rho)$. 
Theorem 5.2. Let $\rho$ be a spectral measure as above and $f \in \ell_{2}^{H}(\{1, \ldots, N\})$ for some $N \in \mathbb{N}$. Then

$$
\|U f\|_{L_{2}(\mathbb{R}, d \rho)} \leq\|f\|_{\ell_{2}^{H}} .
$$

This of course implies that $U$ has a unique continuous extension to all of $\ell_{2}^{H}(\mathbb{N})$, and this extension (which we also denote by $U$ ) still satisfies (5.1).

Proof. Fix $f \in \ell_{2}^{H}(\{1, \ldots, N\})$. Let $\phi$ be a continuous function on $\mathbb{R}$ with compact support, $0 \leq \phi \leq 1$, and $\phi(0)=1$. As discussed in Sect. $3, U$ is a contraction (in fact, a partial isometry) from $\ell_{2}^{H}\left(\left\{1, \ldots, N^{\prime}\right\}\right)$ onto $L_{2}\left(\mathbb{R}, d \rho_{\beta^{\prime}}^{N^{\prime}}\right)$ for every $N^{\prime} \in \mathbb{N}$. Hence if $N_{j} \geq N$ and $R>0$, then

$$
\|f\|_{\ell_{2}^{H}}^{2} \geq \int_{\mathbb{R}}(U f)^{*}(\lambda) d \rho_{\beta_{j}}^{\left(N_{j}\right)}(\lambda)(U f)(\lambda) \geq \int_{\mathbb{R}} \phi\left(\frac{\lambda}{R}\right)(U f)^{*}(\lambda) d \rho_{\beta_{j}}^{\left(N_{j}\right)}(\lambda)(U f)(\lambda) .
$$

Letting $j \rightarrow \infty$ shows that

$$
\|f\|_{\ell_{2}^{H}}^{2} \geq \int_{\mathbb{R}} \phi\left(\frac{\lambda}{R}\right)(U f)^{*}(\lambda) d \rho(\lambda)(U f)(\lambda),
$$

and now (5.1) follows by letting $R \rightarrow \infty$.

Theorem 5.2 has the following important corollary.

Theorem 5.3. Let $\rho$ be a spectral measure of the half line problem. Denote the orthogonal projection onto $R\left(H_{n}\right) \subset \mathbb{C}^{2 d}$ by $P_{n}$. Then for every $n \in \mathbb{N}$,

$$
\int_{\mathbb{R}} H_{n}^{1 / 2} u(n, \lambda) d \rho(\lambda) u^{*}(n, \lambda) H_{n}^{1 / 2} \leq P_{n} .
$$

Proof. Identical to the proof of Theorem 3.4, with Corollary 3.3 replaced by Theorem 5.2

Our second major goal in this section is to prove an invariance result for the absolutely continuous parts of the spectral measures if the boundary condition at $n=1$ is varied. To this end, we will first establish a transformation formula for the $M$ functions. We need the following technical result.

Lemma 5.4. Suppose that $\mu_{N} \rightarrow \mu$ in the weak $*$ topology. Then there is a subsequence $N_{j} \rightarrow \infty$, so that the corresponding functions $M_{N_{j}}$ (compare (4.4)) converge locally uniformly on $\mathbb{C}^{+}$. The limit function has the Herglotz representation

$$
M(z)=A+\int_{\mathbb{R}} \frac{1+t z}{t-z} d \mu(t) .
$$

It is in fact well known (see [5]) that weak $*$ convergence of the measures is equivalent to locally uniform convergence of the imaginary parts of the corresponding Herglotz functions. Here, the real parts can be made convergent too because of the Weyl geometry. With the use of this term, we are referring to the fact that the sets $\mathcal{D}_{N}(z)$ are nested.

Proof. The integrals from the Herglotz representation (4.4) clearly converge locally uniformly. Moreover, $A_{N}=\operatorname{Re} M_{N}(i)$ remains bounded because $M_{N}(i) \in \mathcal{D}_{1}(i)$ for all $N \in \mathbb{N}$, and thus $A_{N}$ converges on a suitable subsequence. 
From now on, we will make the additional assumption that

$$
\bigcap_{n \in \mathbb{N}} N\left(H_{n}\right)=\{0\} \text {. }
$$

Equivalently, if $y$ solves $J\left(y_{n+1}-y_{n}\right)=z H_{n} y_{n}$ for some $z \in \mathbb{C}$ and $\|y\|_{\ell_{2}^{H}}=0$, then $y_{n} \equiv 0$.

We want to analyze the effects of a change of boundary conditions at $n=1$. Let $\alpha$ and $\gamma$ be such boundary conditions. Suppose that $\mu_{\alpha, \beta_{j}}^{\left(N_{j}\right)} \rightarrow \mu_{\alpha}, \mu_{\gamma, \beta_{j}}^{\left(N_{j}\right)} \rightarrow \mu_{\gamma}$ in the weak $*$ topology. By Lemma 5.4 we may assume that the corresponding $M$ functions also converge (locally uniformly) to the limits $M_{\alpha}$ and $M_{\gamma}$, respectively.

Lemma 5.5. The following transformation formula holds for all $z \in \mathbb{C}^{+}$:

$$
M_{\alpha}(z)=\left(-\delta_{2}+\delta_{1} M_{\gamma}(z)\right)\left(\delta_{1}+\delta_{2} M_{\gamma}(z)\right)^{-1},
$$

where

$$
\delta_{1}=\alpha_{1} \gamma_{1}^{*}+\alpha_{2} \gamma_{2}^{*}, \quad \delta_{2}=\alpha_{2} \gamma_{1}^{*}-\alpha_{1} \gamma_{2}^{*} .
$$

Proof. The corresponding formula for the $M$ functions on finite intervals,

$$
M_{\alpha, \beta_{j}}^{\left(N_{j}\right)}(z)=\left(-\delta_{2}+\delta_{1} M_{\gamma, \beta_{j}}^{\left(N_{j}\right)}(z)\right)\left(\delta_{1}+\delta_{2} M_{\gamma, \beta_{j}}^{\left(N_{j}\right)}(z)\right)^{-1}
$$

follows from a straightforward computation (one just has to relate the fundamental matrices $Y_{\alpha}, Y_{\gamma}$ ), which we leave to the reader. Thus it suffices to show that $\delta_{1}+\delta_{2} M_{\gamma}(z)$ is invertible for all $z \in \mathbb{C}^{+}$, for we can then let $j \rightarrow \infty$ in (5.2). So fix $z \in \mathbb{C}^{+}$and suppose that

$$
\left(\delta_{1}+\delta_{2} M_{\gamma}(z)\right) v=0 .
$$

We can also write this in the form

$$
\left(\alpha_{1}, \alpha_{2}\right)\left(\begin{array}{cc}
\gamma_{1}^{*} & -\gamma_{2}^{*} \\
\gamma_{2}^{*} & \gamma_{1}^{*}
\end{array}\right)\left(\begin{array}{c}
1 \\
M_{\gamma}(z)
\end{array}\right) v=0
$$

We abbreviate $F=F_{M_{\gamma}}^{(\gamma)}$; that is, $F(n, z)=Y_{\gamma}(n, z)\left(\begin{array}{c}1 \\ M_{\gamma}(z)\end{array}\right)$ and, similarly,

$$
F_{j}(n, z)=Y_{\gamma}(n, z)\left(\begin{array}{c}
1 \\
M_{\gamma, \beta_{j}}^{\left(N_{j}\right)}(z)
\end{array}\right) \text {. }
$$

Since $F_{j}$ satisfies the boundary condition $\beta_{j}$ at $n=N_{j}+1$, Green's identity (2.1) implies that

$$
2 i \operatorname{Im} z v^{*} \sum_{n=1}^{N_{j}} F_{j}^{*}(n, z) H(n) F_{j}(n, z) v=-\left(F_{j}(1, z) v\right)^{*} J F_{j}(1, z) v .
$$

Clearly, $F_{j}(1, z) \rightarrow F(1, z)$ as $j \rightarrow \infty$, and (5.3) says that $\left(\alpha_{1}, \alpha_{2}\right) F(1, z) v=0$; hence

$$
\lim _{j \rightarrow \infty} v^{*} \sum_{n=1}^{N_{j}} F_{j}^{*}(n, z) H(n) F_{j}(n, z) v=0 .
$$

The summands are non-negative and

$$
\lim _{j \rightarrow \infty} v^{*} F_{j}(n, z) H(n) F_{j}(n, z) v=v^{*} F(n, z) H(n) F(n, z) v .
$$


Therefore, this limit must be equal to zero: $H(n) F(n, z) v=0$ for all $n \in \mathbb{N}$. So $f=F v$ solves $J\left(f_{n+1}-f_{n}\right)=z H_{n} f_{n}$ and represents the zero element of $\ell_{2}^{H}$. Thus, by our assumption, $F(n, z) v \equiv 0$. In particular, $F(1, z) v=0$; hence

$$
\left(\gamma_{1}^{*}-\gamma_{2}^{*} M_{\gamma}(z)\right) v=0, \quad\left(\gamma_{2}^{*}+\gamma_{1}^{*} M_{\gamma}(z)\right) v=0 .
$$

Multiply the first equation from the left by $\gamma_{1}$, multiply the second equation by $\gamma_{2}$ and take the sum. It follows that $v=0$.

Theorem 5.6. Let $\mu_{\alpha}$ and $\mu_{\gamma}$ be the weak $*$ limits of $\mu_{\alpha, \beta_{j}}^{\left(N_{j}\right)}$ and $\mu_{\gamma, \beta_{j}}^{\left(N_{j}\right)}$, respectively. Then the absolutely continuous parts of $\mu_{\alpha}$ and $\mu_{\gamma}$ are equivalent.

The absolutely continuous parts are equivalent in the following strong sense ("with multiplicities"): Write $d \mu_{\alpha, a c}(\lambda)=F_{\alpha}(\lambda) d \lambda, d \mu_{\gamma, a c}(\lambda)=F_{\gamma}(\lambda) d \lambda$, where the densities $F$ take values in the set of non-negative $d \times d$ matrices. Put, for $m=0,1, \ldots, d$,

$$
S_{m}^{(\theta)}=\left\{\lambda \in \mathbb{R}: \operatorname{rank} F_{\theta}(\lambda)=d\right\} \quad(\theta=\alpha, \gamma) .
$$

Then the symmetric difference $S_{m}^{(\alpha)} \Delta S_{m}^{(\gamma)}$ has Lebesgue measure zero for all $m=$ $1, \ldots, d$.

Proof. Gesztesy and Tsekanovskii prove that this result follows from the transformation formula for the $M$ functions from Lemma [5.5. See [7, Theorem 6.6].

\section{Symplectic Linear algebra}

Let $V$ be a complex vector space, and let $\omega$ be a sesquilinear form on $V$ with $\omega(v, w)=-\overline{\omega(w, v)}$. If $\omega(v, w)=0 \forall w \in V$ implies $v=0, V$ is called a (complex) symplectic linear space, and $\omega$ is called a symplectic form. Symplectic spaces play an important role in the analysis of canonical systems because the boundary form $\omega(u, v):=u^{*} J v$ is a symplectic form on $\mathbb{C}^{2 d}$.

One is often more interested in real symplectic linear spaces (and real symplectic manifolds) because of their fundamental role in the mathematical formulation of classical mechanics. See [11, Chapter 1, §1] and [12, Chapter 1, Sect. 2]. For a use of the methods of complex symplectic linear algebra in the theory of differential operators, see 6 .

The main results of this section are Theorem 6.6 and Corollary 6.7. This latter result will play a crucial role in the next section. Roughly speaking, it says that there are so-called Lagrangian subspaces (subspaces that correspond to self-adjoint boundary conditions) in many different directions.

For $W \subset V$, let $W^{\omega}=\{v \in V: \omega(v, w)=0 \forall w \in W\}$. Clearly, $W^{\omega}$ is a subspace of $V$. A subspace $W$ of $V$ is called isotropic if $W \subset W^{\omega}$, and it is called symplectic if $W \cap W^{\omega}=\{0\}$.

We will only deal with finite-dimensional symplectic spaces $V$. We then have:

Lemma 6.1. Let $W$ be a subspace of $V$. Then

$$
\operatorname{dim} W+\operatorname{dim} W^{\omega}=\operatorname{dim} V, \quad W^{\omega \omega}=W .
$$


Proof. The map $A: V \rightarrow V^{\prime},(A v)(w)=\omega(v, w)$ is an (anti-linear) isomorphism because ker $A=\{0\}$. Hence

$$
\operatorname{dim} W^{\omega}=\operatorname{dim} A W^{\omega}=\operatorname{dim}\left\{F \in V^{\prime}: F(v)=0 \forall v \in W\right\}=\operatorname{dim} V-\operatorname{dim} W .
$$

To prove the second assertion, note that $W \subset W^{\omega \omega}$ by the definition of $(\cdots)^{\omega}$. On the other hand, by what has been proved already,

$$
\operatorname{dim} W^{\omega \omega}=\operatorname{dim} V-\operatorname{dim} W^{\omega}=\operatorname{dim} W,
$$

hence $W=W^{\omega \omega}$.

The following is an immediate consequence of Lemma 6.1.

Corollary 6.2. Let $W$ be a symplectic subspace of $V$. Then $W^{\omega}$ is a symplectic subspace and $V=W+W^{\omega}$.

Lemma 6.3. Let $V$ be a symplectic space with $\operatorname{dim} V=D$. Then there exists an isomorphism $\psi: V \rightarrow \mathbb{C}^{D}$ so that

$$
\omega(v, w)=\psi(v)^{*} i\left(\begin{array}{cc}
1_{p} & 0 \\
0 & -1_{q}
\end{array}\right) \psi(w) .
$$

Proof. If $V$ is identified with $\mathbb{C}^{D}$, the symplectic form takes the form $\omega(v, w)=$ $v^{*} A w$ with $i A$ self-adjoint and invertible. A diagonalization of $A$ and then a further transformation with a diagonal matrix yields the asserted form of $\omega$.

The number $p \in\{0,1, \ldots, D\}$ from the lemma characterizes the symplectic space $V$; it is an invariant under symplectomorphisms (linear isomorphisms that preserve the symplectic form). Obviously, $q=D-p$.

The simple normal form of $\omega$ from Lemma 6.3 also shows that we can find a basis $B=\left\{e_{1}, \ldots, e_{p}, f_{1}, \ldots, f_{q}\right\}$ of $V$ so that $\omega\left(e_{j}, e_{k}\right)=i \delta_{j k}, \omega\left(f_{j}, f_{k}\right)=-i \delta_{j k}$, and $\omega\left(e_{j}, f_{k}\right)=0$. A basis satisfying these conditions will be called a symplectic basis.

Lemma 6.4. Let $W$ be a subspace of the symplectic space $V$. Then $W /\left(W \cap W^{\omega}\right)$ is a symplectic space.

Here, we define a symplectic form on the quotient by $\omega((v),(w))=\omega(v, w)$. This makes sense because obviously the right-hand side is independent of the choice of the representatives.

Proof. It is obvious that the form defined above is sesquilinear on the quotient and satisfies $\omega((v),(w))=-\overline{\omega((w),(v))}$. If $\omega((v),(w))=0$ for all $(w) \in W /\left(W \cap W^{\omega}\right)$, then $\omega(v, w)=0$ for all $w \in W$. This means that $v \in W^{\omega}$ or $(v)=0$.

Lemma 6.5. Let $W$ be an isotropic subspace of $V$ with $\operatorname{dim} W=k$. Then there exists an isotropic subspace $Z$ with $\operatorname{dim} Z=k$ and $W \cap Z=\{0\}$ so that $W \dot{+} Z$ is a symplectic subspace of $V$.

Proof. We may assume that $V=\mathbb{C}^{D}$ and $\omega$ has the form given in Lemma 6.3. Write $A=i\left(\begin{array}{cc}1_{p} & 0 \\ 0 & -1_{q}\end{array}\right)$ and put $Z=A W$. Then $\operatorname{dim} Z=\operatorname{dim} W=k$ and $Z$ is isotropic because $A^{*}=-A, A^{2}=-1$ and hence $(A v)^{*} A(A w)=v^{*} A w=0$ for all $v, w \in W$. If $v \in W \cap Z$, then $v^{*} v=v^{*} A\left(A^{-1} v\right)=0$, hence $v=0$. 
It remains to show that $W \dot{+} Z$ is symplectic. Suppose that $v=v_{1}+A v_{2}\left(v_{j} \in W\right)$ has the property that $v^{*} A w=0$ for all $w=w_{1}+A w_{2}$ with $w_{j} \in W$. By multiplying out and using the fact that $W$ and $Z$ are isotropic, we see that then $v_{2}^{*} w_{1}=v_{1}^{*} w_{2}$ for all $w_{1}, w_{2} \in W$. Since we may in particular take one of the $w_{j}$ 's equal to zero, it follows that $v_{1}=v_{2}=0$, and thus $W \dot{+} Z$ is symplectic.

We will now concentrate on the case $D=2 d$ and $p=q=d$. The motivation for concentrating on this special case is clear: These are the parameters of the boundary form $u^{*} J v$. A subspace $L \subset \mathbb{C}^{2 d}$ is called a Lagrangian subspace if $L=L^{\omega}$. Lagrangian subspaces are precisely the maximal isotropic subspaces. Indeed, if $L=L^{\omega}$, then $L$ clearly is an isotropic subspace that is maximal. Conversely, if $L$ is isotropic with $\operatorname{dim} L=k$, then, by Lemma 6.5 there exists another isotropic subspace $\widetilde{L}$ so that $L \dot{+} \widetilde{L}$ is a $2 k$-dimensional symplectic space. The parameters $p_{0}, q_{0}$ of this subspace must satisfy $p_{0}=q_{0}=k$, for otherwise there could not be a $k$-dimensional isotropic subspace. By Corollary 6.2, $(L \dot{+} \widetilde{L})^{\omega}$ is a direct summand for $L \dot{+} \widetilde{L}$. The parameters of this new subspace are $p_{1}=q_{1}=d-k$, and hence there are isotropic subspaces. It follows that $L$ is not maximal unless $k=d$. But in this case, $\operatorname{dim} L=\operatorname{dim} L^{\omega}$ by Lemma 6.1 and hence $L=L^{\omega}$, as desired.

Theorem 6.6. There exist finitely many Lagrangian subspaces $L_{1}, \ldots, L_{n}$ of $\mathbb{C}^{2 d}$ so that for any d-dimensional subspace $V \subset \mathbb{C}^{2 d}$, we have that $V \dot{+} L_{j}=\mathbb{C}^{2 d}$ for some $j \in\{1, \ldots, n\}$.

Proof. We first prove an apparently weaker version of the theorem: For every $d$ dimensional subspace $V$, there exists a Lagrangian subspace $L$ so that $V \dot{+} L=\mathbb{C}^{2 d}$. It will then be shown by a compactness argument that actually finitely many $L$ 's suffice.

We begin with the special case where $V$ is a symplectic subspace. We fix symplectic bases $\left\{e_{1}, \ldots, e_{j}, f_{1}, \ldots, f_{k}\right\}$ and $\left\{e_{1}^{\prime}, \ldots, e_{j^{\prime}}^{\prime}, f_{1}^{\prime}, \ldots, f_{k^{\prime}}^{\prime}\right\}$ of $V$ and $V^{\omega}$, respectively. We have that $j+k=j^{\prime}+k^{\prime}=d$ and $j+j^{\prime}=k+k^{\prime}=d$. This implies that $j=k^{\prime}, j^{\prime}=k$. Thus we can define

$$
L=\operatorname{span}\left(e_{1}+f_{1}^{\prime}, \ldots, e_{j}+f_{j}^{\prime}, e_{1}^{\prime}+f_{1}, \ldots, e_{j^{\prime}}^{\prime}+f_{j^{\prime}}\right) .
$$

Then $L$ is a $d$-dimensional isotropic (hence Lagrangian) subspace with $V \cap L=\{0\}$.

We may now assume that $V$ is not symplectic. We give a proof by induction on $d$. For $d=1, V=L(v)$ with $\omega(v, v)=0$ (since $V$ is not symplectic). Hence $V$ is isotropic and the existence of a Lagrangian direct summand follows from Lemma 6.5 .

Now suppose that $d \geq 2$. Lemma 6.4 shows that there is a symplectic subspace $S \subset V$ so that $V=V \cap V^{\omega} \dot{+} S$. In particular, $V \cap V^{\omega} \subset S^{\omega}$. Since $V$ is not symplectic, $k:=\operatorname{dim} V \cap V^{\omega} \geq 1$. By Lemma 6.5, applied to the isotropic subspace $V \cap V^{\omega}$ of the symplectic space $S^{\omega}$, there is an isotropic subspace $I \subset S^{\omega}$ with $\operatorname{dim} I=k$, so that $K:=V \cap V^{\omega} \dot{+} I$ is a symplectic subspace of $S^{\omega}$. Let $T=V \cap K^{\omega}$. Then, since $S \subset K^{\omega}$,

$$
V=V \cap V^{\omega} \dot{+} S \subset V \cap V^{\omega} \dot{+} V \cap K^{\omega}=V \cap V^{\omega} \dot{+} T \subset V,
$$

and hence $V=V \cap V^{\omega} \dot{+} T$. Note that the sum is indeed direct because $K \cap K^{\omega}=$ $\{0\}$. In particular,

$$
\operatorname{dim} T=\operatorname{dim} V-\operatorname{dim}\left(V \cap V^{\omega}\right)=d-k=\frac{1}{2} \operatorname{dim} K^{\omega} .
$$


Since $K$ is a $2 k$-dimensional symplectic space that has a $k$-dimensional isotropic subspace (namely, $I$ ), it follows that the parameters from Lemma 6.3 are $p_{K}=$ $q_{K}=k$. Thus also $p_{K^{\omega}}=q_{K^{\omega}}=d-k$, and, recalling (6.1), we may apply the induction hypothesis to the symplectic space $K^{\omega}$ and the subspace $T$. We obtain an isotropic subspace $J \subset K^{\omega}$ so that $T \dot{+} J=K^{\omega}$.

Let $L=I \dot{+} J$ (the sum is direct because $I \subset K$ and $J \subset K^{\omega}$ ). Then

$$
V+L=V \cap V^{\omega} \dot{+} T+(I \dot{+} J)=\left(V \cap V^{\omega}+I\right)+(T+J)=K \dot{+} K^{\omega}=\mathbb{C}^{2 d} .
$$

Moreover, $L$ is isotropic. This concludes the proof of the simplified version of the theorem.

To prove that finitely many $L$ 's suffice, we work with the (complex) Grassmannian $G_{d, 2 d}$, the manifold of $d$-dimensional subspaces of $\mathbb{C}^{2 d}$. The crucial fact is that $G_{d, 2 d}$ is a compact space in natural topology. See, for example, [13, Lemma 5.1].

Moreover, if $L \cap V_{0}=\{0\}$ for some $V_{0} \in G_{d, 2 d}$, then in fact $L \cap V=\{0\}$ for all $V$ from a neighborhood of $V_{0}$. Thus the compactness of $G_{d, 2 d}$ now gives the full claim of the theorem.

Corollary 6.7. Let $L_{1}, \ldots, L_{n}$ be as in Theorem 6.6, and let $S_{j} \subset L_{j}(j=1, \ldots, n)$ be $m$-dimensional subspaces of these spaces $(m \geq 1)$. Then

$$
\operatorname{dim} \operatorname{span}\left(S_{1}, \ldots, S_{n}\right) \geq d+m .
$$

Proof. Write $S=\operatorname{span}\left(S_{1}, \ldots, S_{n}\right)$ and let $V$ be a $k$-dimensional subspace of $S$ with $k \leq d$. By Theorem 6.6. $V \cap L_{j}=\{0\}$ for some $j$ and hence $S$ has a $(k+m)$ dimensional subspace. The assertion follows by iterating this argument.

We conclude this section by establishing the characterization of Lagrangian subspaces that has already been used in Sect. 2 and the following sections.

Theorem 6.8. $L$ is a Lagrangian subspace of $\mathbb{C}^{2 d}$ with symplectic form $\omega(u, v)=$ $u^{*} J v$ if and only if there are $\alpha_{1}, \alpha_{2} \in \mathbb{C}^{d \times d}$ satisfying (2.2) so that

$$
L=\left\{v \in \mathbb{C}^{2 d}:\left(\alpha_{1}, \alpha_{2}\right) v=0\right\}
$$

Proof. Given a Lagrangian subspace $L$, choose an orthonormal basis of $L$ to represent $L$ as

$$
L=\left\{\left(\begin{array}{c}
-\alpha_{2}^{*} \\
\alpha_{1}^{*}
\end{array}\right) c: c \in \mathbb{C}^{d}\right\}
$$

where $\alpha_{1} \alpha_{1}^{*}+\alpha_{2} \alpha_{2}^{*}=1$ (this condition says that the columns of $\left(\begin{array}{c}-\alpha_{2}^{*} \\ \alpha_{1}^{*}\end{array}\right)$ form an orthonormal system). Then, as $L$ is isotropic, we must have that

$$
\left(-\alpha_{2}, \alpha_{1}\right) J\left(\begin{array}{c}
-\alpha_{2}^{*} \\
\alpha_{1}^{*}
\end{array}\right)=\alpha_{2} \alpha_{1}^{*}-\alpha_{1} \alpha_{2}^{*}=0 .
$$

So, first of all, the $\alpha$ 's satisfy (2.2). It now also follows that $L$ has the alternate description (6.2). 
These steps may be reversed: If, conversely, $L$ is given by (6.2) with $\alpha$ 's satisfying (2.2), then $L$ may also be described by (6.3) and hence is Lagrangian.

\section{Proof of Theorem 1.1}

We now have all the tools for the proof of Theorem 1.1. The set $S_{m}$ was defined in (5.4). Here, we can just fix an arbitrary boundary condition at $n=1$. Then $S_{m}$ for a different boundary condition differs from this fixed set by a set of measure zero which is clearly irrelevant because Theorem 1.1 asserts the existence of (weakly) bounded solutions only almost everywhere on $S_{m}$.

Theorem 1.1 will be a consequence of the following result.

Theorem 7.1. There exists a function $f>0$ on $S_{m}$ and, for every $\lambda \in S_{m}, a$ subspace $V_{\lambda} \subset \mathbb{C}^{2 d}$ with $\operatorname{dim} V_{\lambda}=m$, so that

$$
\int_{S_{m}} v(\lambda)^{*} Y(n, \lambda)^{*} H(n) Y(n, \lambda) v(\lambda) f(\lambda) d \lambda \leq 1
$$

for every $n \in \mathbb{N}$ and every measurable choice $v(\lambda) \in V_{\lambda}$ with $\|v(\lambda)\|=1$.

Proof. By definition of $S_{m}, \chi_{S_{m}}(\lambda) d \rho_{a c}(\lambda)=F(\lambda) d \lambda$, where $F(\lambda) \in \mathbb{C}^{d \times d}$ has precisely $m$ positive eigenvalues. The remaining $d-m$ eigenvalues (counting multiplicities) are equal to zero. Let $f(\lambda)$ be the smallest positive eigenvalue, and let $V_{\lambda} \subset \mathbb{C}^{d}$ be the span of the eigenvectors corresponding to positive eigenvalues.

We will now use Theorem 5.3. This result implies that

$$
\int_{S_{m}} H_{n}^{1 / 2} u(n, \lambda) d \rho(\lambda) u^{*}(n, \lambda) H_{n}^{1 / 2} \leq 1 .
$$

Since $\rho(M) \geq \rho_{a c}(M) \geq \int_{M} P_{\lambda} f(\lambda) d \lambda$, where $P_{\lambda}$ is the orthogonal projection onto $V_{\lambda}$, we have that

$$
\int_{S_{m}} H_{n}^{1 / 2} u(n, \lambda) v(\lambda)\left(H_{n}^{1 / 2} u(n, \lambda) v(\lambda)\right)^{*} f(\lambda) d \lambda \leq 1
$$

for all measurable choices $v(\lambda) \in V_{\lambda}$ with $\|v(\lambda)\| \equiv 1$. Of course, this inequality holds in the sense of positive definiteness (and $1 \in \mathbb{C}^{2 d \times 2 d}$ ). Hence for every unit vector $e \in \mathbb{C}^{2 d}$, we have that

$$
\int_{S_{m}} e^{*} H_{n}^{1 / 2} u(n, \lambda) v(\lambda)\left(H_{n}^{1 / 2} u(n, \lambda) v(\lambda)\right)^{*} e f(\lambda) d \lambda \leq 1 .
$$

Here, however, $e^{*} H_{n}^{1 / 2} u(n, \lambda) v(\lambda)$ is a complex number; thus we can change the order to obtain

$$
\int_{S_{m}} v(\lambda)^{*} u(n, \lambda)^{*} H_{n}^{1 / 2} e e^{*} H_{n}^{1 / 2} u(n, \lambda) v(\lambda) f(\lambda) d \lambda \leq 1 .
$$

Note that $e e^{*}$ is the projection onto $L(e)$; thus summing over an orthonormal basis gives

$$
\int_{S_{m}} v(\lambda)^{*} u(n, \lambda)^{*} H_{n} u(n, \lambda) v(\lambda) f(\lambda) d \lambda \leq 2 d .
$$


This is the assertion of the theorem, as $Y=(\ldots, u)$, and we can thus simply disregard the first $d$ columns of $Y$. Finally, we can of course get the constant 1 on the right-hand side by adjusting $f$.

Theorem 1.1 is now obtained as follows. Fix Lagrangian subspaces (or, equivalently, boundary conditions) as in Corollary 6.7. Apply Theorem 7.1 with these boundary conditions at $n=1$ (although it has not been made explicit in the notation, we worked with a fixed but arbitrary boundary condition in this theorem). Of course, we may replace $f_{\alpha}(\lambda)$ by the minimum of these functions over the boundary conditions $\alpha$ we are considering. This new function will again be denoted by $f$, without index.

Fatou's Lemma shows that for every boundary condition $\alpha$, there is a set $N_{\alpha}$ of zero Lebesgue measure, so that

$$
\liminf _{j \rightarrow \infty} v^{*} Y^{*}\left(n_{j}, \lambda\right) H\left(n_{j}\right) Y\left(n_{j}, \lambda\right) v<\infty
$$

if $\lambda \in S_{m} \backslash N_{\alpha}$ and $v \in V_{\lambda}^{(\alpha)}$. Here, we get rid of the $\lambda$ dependence of $v(\lambda)$ from Theorem 7.1 by making $m$ special choices $v(\lambda)$ in such a way that the $m$ different $v(\lambda)$ 's span $V_{\lambda}$ for every $\lambda \in S_{m}$. Also, we use the same fundamental matrix $Y$, let us say the one with $Y(1, \lambda)=1$, for all boundary conditions. This can be done since a change of $Y$ just amounts to a transformation of the spaces $V_{\lambda}^{(\alpha)}$.

Clearly, condition (7.1) defines a subspace of vectors $v \in \mathbb{C}^{2 d}$. Put more abstractly, we have thus shown that to each of the boundary conditions (Lagrangian subspaces) chosen above, there corresponds an at least $m$-dimensional subspace of this Lagrangian subspace on which (7.1) holds. This $m$-dimensional space really is a subspace of the Lagrangian subspace because the solutions $Y(\cdot, \lambda) v$ constructed above satisfy the given boundary condition at $n=1$. This in turn follows from the proof of Theorem [7.1, where these solutions were in fact obtained as linear combinations of the columns of $u_{\alpha}(\cdot, \lambda)$.

Summing up, we see that we are in the situation of Corollary 6.7. This result shows that the space of vectors $v \in \mathbb{C}^{2 d}$ satisfying (7.1) is of dimension at least $d+m$, and this is exactly what Theorem 1.1 states.

\section{Higher ORDER EQUATIONS}

In this section, we show that any formally self-adjoint equation of even order $2 d$ can be written as a canonical system. This is a rather comforting fact because it shows that canonical systems indeed provide a very general framework. The canonical system formulation in fact has the advantage that it automatically (almost inadvertently) handles correctly some somewhat subtle issues related to the reduction of the Hilbert space for certain boundary conditions.

Our starting point is the difference equation

$$
\sum_{j=1}^{d}\left(c_{j}(n+j) y(n+j)+c_{j}(n) y(n-j)\right)+c_{0}(n) y(n)=z w(n) y(n) .
$$

The coefficients $c_{j}$ are real valued, and $c_{d}(n) \neq 0, w(n)>0$ for all $n$. We can write (8.1) formally as $\tau y=z y$, where $(\tau y)(n)$ is given by the left-hand side of (8.1), divided by $w(n)$. As is well known, the difference expression $\tau$ generates self-adjoint operators in the Hilbert spaces $\ell_{2}^{w}$ with scalar product $\langle f, g\rangle=\sum \overline{f(n)} w(n) g(n)$. 
To write (8.1) as a canonical system, we introduce the vector $Y(n) \in \mathbb{C}^{2 d}$ by

$$
Y_{k}(n)= \begin{cases}y(n+k-d-1) & (k=1, \ldots, d), \\ -\sum_{j=1}^{k-d} c_{2 d-k+j}(n-1+j) y(n-1+j) & (k=d+1, \ldots, 2 d) .\end{cases}
$$

Lemma 8.1 (Green's identity).

$$
\sum_{n=1}^{N}(\overline{f(n)} w(n)(\tau g)(n)-\overline{(\tau f)(n)} w(n) g(n))=F^{*}(N+1) J G(N+1)-F^{*}(1) J G(1) .
$$

Here, $F, G$ are obtained from $f, g$ as in (8.2).

Proof. This follows from a computation:

$$
\begin{aligned}
\sum_{n=1}^{N} & \overline{f(n)} w(n)(\tau g)(n) \\
= & \sum_{n=1}^{N} \overline{f(n)}\left[\sum_{j=1}^{d}\left(c_{j}(n+j) g(n+j)+c_{j}(n) g(n-j)\right)+c_{0}(n) g(n)\right] \\
= & \sum_{j=1}^{d} \sum_{n=j+1}^{N+j} \overline{f(n-j)} c_{j}(n) g(n)+\sum_{j=1}^{d} \sum_{n=1-j}^{N-j} \overline{f(n+j)} c_{j}(n+j) g(n) \\
& +\sum_{n=1}^{N} \overline{f(n)} c_{0}(n) g(n) \\
= & \sum_{n=1}^{N} \overline{(\tau f)(n)} w(n) g(n) \\
& +\sum_{j=1}^{d} \overline{\sum_{n=N+1}^{N+j}} \overline{f(n-j)} c_{j}(n) g(n)-\sum_{j=1}^{d} \sum_{n=1}^{j} \overline{f(n-j)} c_{j}(n) g(n) \\
& -\sum_{j=1}^{d} \sum_{n=N-j+1}^{N} \overline{f(n+j)} c_{j}(n+j) g(n)+\sum_{j=1}^{d} \sum_{n=1-j}^{0} \overline{f(n+j)} c_{j}(n+j) g(n) .
\end{aligned}
$$

To establish the lemma, we will now show that

$$
\begin{aligned}
F^{*}(N+1) J G(N+1)= & \sum_{j=1}^{d} \sum_{n=N+1}^{N+j} \overline{f(n-j)} c_{j}(n) g(n) \\
& -\sum_{j=1}^{d} \sum_{n=N-j+1}^{N} \overline{f(n+j)} c_{j}(n+j) g(n), \\
F^{*}(1) J G(1)= & \sum_{j=1}^{d} \sum_{n=1}^{j} \overline{f(n-j)} c_{j}(n) g(n) \\
& -\sum_{j=1}^{d} \sum_{n=1-j}^{0} \overline{f(n+j)} c_{j}(n+j) g(n) .
\end{aligned}
$$


Let us look at the first sum from (8.3). We have that

$$
\begin{aligned}
\sum_{j=1}^{d} & \sum_{n=N+1}^{N+j} \overline{f(n-j)} c_{j}(n) g(n) \\
& =\sum_{j=1}^{d} \sum_{n=N+1}^{N+j} \overline{F_{n-j+d-N}(N+1)} c_{j}(n) g(n) \\
& =\sum_{j=1}^{d} \sum_{k=d+1-j}^{d} \overline{F_{k}(N+1)} c_{j}(N+j-d+k) g(N+j-d+k) \\
& =\sum_{k=1}^{d} \overline{F_{k}(N+1)} \sum_{j=d+1-k}^{d} c_{j}(N+j-d+k) g(N+j-d+k) \\
& =\sum_{k=1}^{d} \overline{F_{k}(N+1)} \sum_{j=1}^{k} c_{j+d-k}(N+j) g(N+j)=-\sum_{k=1}^{d} \overline{F_{k}(N+1)} G_{d+k}(N+1) .
\end{aligned}
$$

As for the second sum from (8.3), a similar calculation shows that

$$
-\sum_{j=1}^{d} \sum_{n=N-j+1}^{N} \overline{f(n+j)} c_{j}(n+j) g(n)=\sum_{k=1}^{d} \overline{F_{d+k}(N+1)} G_{k}(N+1) .
$$

These formulae prove (8.3). A similar computation works for (8.4).

The difference equation (8.1) is equivalent to a first order difference equation for the vector $Y: Y(n+1, z)=(z A(n)+B(n)) Y(n, z)$, where

$$
\begin{gathered}
A_{2 d, d+1}(n)=\frac{w(n)}{c_{d}(n)}, \\
B_{j, j+1}(n)=1 \quad(j=1, \ldots, d-1, d+1, \ldots, 2 d), \quad B_{d, d+1}(n)=-\frac{1}{c_{d}(n)}, \\
B_{d+j, d+1}(n)=-\frac{c_{d-j}(n)}{c_{d}(n)} \quad(j=1, \ldots, d), \quad B_{2 d, j}(n)=c_{d+1-j}(n) \quad(j=1, \ldots, d),
\end{gathered}
$$

and all other matrix elements are equal to zero.

Let $T(n)$ be the fundamental matrix of this difference equation for $z=0$; that is, $T(n) \in \mathbb{C}^{2 d \times 2 d}, T(1)=1$ and $T(n+1)=B(n) T(n)$. Lemma 8.1 implies that $T^{*}(n) J T(n)=J$; hence $T(n)$ is invertible for every $n$ and we can define $U(n, z)=T^{-1}(n) Y(n, z)$. A straightforward computation shows that $U(n, z)$ solves an equation of the form

$$
J(U(n+1, z)-U(n, z))=z H(n) U(n, z) .
$$

We do not need the explicit form of $H(n)$, but the computation in fact gives that $H(n)=J T(n+1)^{-1} A(n) T(n)$. In particular, the rank of $H(n)$ equals 1.

Since the original equation (8.1) has $2 d$ linearly independent solutions $y(\cdot, z)$ for each fixed $z \in \mathbb{C}$, we get in this way a $2 d$-dimensional space of solutions $U(\cdot, z)$ of (8.5). But the solution space of (8.5) also is of dimension $2 d$, so every solution arises in this way. In other words, if $U$ solves (8.5), then $U=T^{-1} Y$, where $Y$ is defined as in (8.2) and the corresponding $y$ solves (8.1). This solution $y$ is uniquely determined by $U$. So (8.1) and (8.5) are equivalent. 
Of course, (8.5) has the form of a canonical system, but we do not yet know that $H(n)$ has the required properties. We now turn to this question.

Theorem 8.2. $H(n)=H^{*}(n), H(n) \geq 0$ and $H(n) J H(n)=0$ for all $n$. Moreover, $\bigcap_{n=n_{0}}^{n_{0}+2 d-1} N(H(n))=\{0\}$.

Proof. Let $y$ solve (8.1). By Lemma 8.1 .

$$
\begin{aligned}
(z-\bar{z}) \sum_{n=1}^{N}|y(n)|^{2} & =\sum_{n=1}^{N}(\overline{y(n)} w(n)(\tau y)(n)-\overline{(\tau y)(n)} w(n) y(n)) \\
& =Y^{*}(N+1) J Y(N+1)-Y^{*}(1) J Y(1) \\
& =U^{*}(N+1) T^{*}(N+1) J T(N+1) U(N+1)-U^{*}(1) J U(1) \\
& =U^{*}(N+1) J U(N+1)-U^{*}(1) J U(1) .
\end{aligned}
$$

To pass to the last line, we have used the fact that $T^{*}(n) J T(n)=J$. It has already been noted above that this identity follows from Lemma 8.1.

We also have that $|y(n)|^{2}=c_{d}^{-2}(n) Y^{*}(n) P Y(n)$, where $P_{d+1, d+1}=1$ and $P_{i j}=0$ for all other matrix elements. Thus

$$
U^{*}(N+1) J U(N+1)-U^{*}(1) J U(1)=(z-\bar{z}) \sum_{n=1}^{N} U^{*}(n) W(n) U(n),
$$

with $W(n)=c_{d}^{-2}(n) T^{*}(n) P T(n)$. Note that $W(n) \geq 0$.

So far, we have used Green's identity for the original equation (8.1) to compute $\sum|y|^{2}$. Now, we do a similar calculation for the system (8.5):

$$
\begin{aligned}
\sum_{n=1}^{N} & U^{*}(n)\left(\bar{z} H^{*}(n)-z H(n)\right) U(n) \\
= & \sum_{n=1}^{N}\left[(J(U(n+1)-U(n)))^{*} U(n)-U^{*}(n) J(U(n+1)-U(n))\right] \\
= & \sum_{n=1}^{N}\left[\left(U^{*}(n+1)-U^{*}(n)\right) J(U(n+1)-U(n))\right. \\
& \left.\quad-U^{*}(n+1) J U(n+1)+U^{*}(n) J U(n)\right] \\
= & \sum_{n=1}^{N}\left(U^{*}(n+1)-U^{*}(n)\right) J(U(n+1)-U(n)) \\
& \quad-U^{*}(N+1) J U(N+1)+U^{*}(1) J U(1) \\
= & \sum_{n=1}^{N}|z|^{2} U^{*}(n) H^{*}(n) J H(n) U(n)-U^{*}(N+1) J U(N+1)+U^{*}(1) J U(1) .
\end{aligned}
$$

By combining this identity with (8.6), we see that

$$
\sum_{n=1}^{N} U^{*}(n)\left(\bar{z} H^{*}(n)-z H(n)-|z|^{2} H^{*}(n) J H(n)+(z-\bar{z}) W(n)\right) U(n)=0 .
$$

We must recall that, although not indicated in the notation, the solutions $U$ of course also depend on $z$. This dependence is continuous (in fact $U(n, z)$ is a polynomial in $z$ ) if we specialize to solutions with $z$ independent initial values. 
First of all, we choose $z=\epsilon$ real. After dividing by $\epsilon$, we get from (8.7) that

$$
\sum_{n=1}^{N} U^{*}(n, \epsilon)\left(H^{*}(n)-H(n)-\epsilon H^{*}(n) J H(n)\right) U(n, \epsilon)=0 .
$$

Letting $\epsilon \rightarrow 0$ shows that

$$
\sum_{n=1}^{N} U^{*}(n, 0)\left(H^{*}(n)-H(n)\right) U(n, 0)=0 .
$$

This holds for any solution $U(n, 0)$ of $J(U(n+1,0)-U(n, 0))=0$, that is, for any constant vector $U(n, 0)=v, v \in \mathbb{C}^{2 d}$. Moreover, $N$ is also arbitrary, hence $H^{*}(n)=H(n)$, as claimed.

Next, we take $z=i \epsilon$, again with $\epsilon \in \mathbb{R}, \epsilon \neq 0$. In the same way as in the preceding paragraph, we now see that $H(n)=W(n) \geq 0$.

Finally, once we know that $H^{*}(n)=H(n)=W(n)$, we also see with the help of this argument from (8.7) that $H(n) J H(n)=0$.

It remains to show that $\bigcap N(H(n))=\{0\}$. If $H(n) v=0$ for $n=n_{0}, \ldots, n_{0}+$ $2 d-1$, then $U(n):=v$ solves $J(U(n+1)-U(n))=z H(n) U(n)$ for these $n$ (and for all $z$ ). Thus there is a corresponding solution $y$ to (8.1) so that $U(n)=T(n)^{-1} Y(n)$, and now the explicit form of $H(n)=W(n)$ given in the line following (8.6) shows that $P Y(n)=0$ or $y(n)=0$ for $n=n_{0}, \ldots, n_{0}+2 d-1$. This in turn shows that $Y\left(n_{0}+d\right)=0$, so $v=0$.

Note also that since $\operatorname{dim} N(H(n))=2 d-1$, we need an intersection over at least $2 d$ such kernels to get the zero space.

We conclude with a discussion of the role of the space $Z$ from Sect. 2 for the canonical systems from this section. We consider the system (8.5) on $n \in$ $\{1, \ldots, N\}$ with boundary conditions of the form (2.3). Of course, we still assume that (8.5) comes from a higher order equation of the form of (8.1). Then $Z \perp \ell_{2}^{H}(\{d+1, \ldots, N-d\})$. We now sketch the proof of this assertion. First of all, the inhomogeneous equation $\tau y=w f$ is also equivalent to an inhomogeneous canonical system $J(U(n+1)-U(n))=H(n) F(n)$, with $U$ calculated from $y$ in the same way as above, and the relation between $F$ and $f$ is analogous. Now if $H(n) U(n)=0$ for $n=1, \ldots, N$, then also $y(n)=0$ for these $n$. This follows as in the last part of the proof of Theorem 8.2. Consequently, $U(d+1)=\cdots=U(N-d+1)=0$ and hence $H(d+1) F(d+1)=\cdots=H(N-d) F(N-d)=0$, as claimed.

This means that the Hilbert space $\ell_{2}^{H} \ominus Z$ differs from $\ell_{2}^{H}$ only near the endpoints $n=1$ and $n=N$. There are boundary conditions for which the underlying Hilbert space must be modified, and the space $Z$ takes care of precisely this effect. Rather than enter a lengthy general discussion, we will just illustrate this phenomenom with an example.

We consider the difference expression $(\tau y)(n)=y(n-1)+y(n+1)$ on $n \in$ $\{1, \ldots, N\}$. We want to construct self-adjoint operators on $\ell_{2}(\{1, \ldots, N\})$ from $\tau$. Usually, one proceeds as follows. One introduces the additional points $n=0$, $n=N+1$, imposes the boundary conditions

$$
y(0) \sin \alpha+y(1) \cos \alpha=0, \quad y(N) \sin \beta+y(N+1) \cos \beta=0,
$$

computes $y(0)$ and $y(N+1)$ from $y(1)$ and $y(N)$, respectively, with the help of these boundary conditions and puts $\left(H_{\alpha, \beta} y\right)(n)=(\tau y)(n)$. For example, $\left(H_{\pi / 2, \beta} y\right)(1)=$ $y(2)$. 
Clearly, if $\alpha=0$ or $\beta=0$, this recipe must be modified. If, let us say, $\alpha=0$, the boundary condition says that $y(1)=0$, and this suggests that we consider $\tau$ on the reduced Hilbert space $\ell_{2}(\{2, \ldots, N\})$.

Let us now see what the canonical system approach gives in this case. For simplicity, we use the boundary condition $y(N+1)=0$ at the right endpoint. From (8.2), we obtain $Y(n)=\left(\begin{array}{c}y(n-1) \\ -y(n)\end{array}\right)$. The boundary condition corresponding to $y(1)=0$ thus is $(0,1) U(1)=0$ (recall that $Y(1)=U(1))$. One also checks easily that

and

$$
T(2 n)=\left(\begin{array}{cc}
0 & -1 \\
-1 & 0
\end{array}\right), \quad T(2 n+1)=\left(\begin{array}{ll}
1 & 0 \\
0 & 1
\end{array}\right)
$$

$$
H(2 n)=\left(\begin{array}{ll}
1 & 0 \\
0 & 0
\end{array}\right), \quad H(2 n+1)=\left(\begin{array}{ll}
0 & 0 \\
0 & 1
\end{array}\right) .
$$

Now a straightforward investigation of the equation $J\left(U_{n+1}-U_{n}\right)=H_{n} F_{n}$, together with the boundary conditions and the condition that $H_{n} U_{n}=0$, shows that $Z$ is the one-dimensional space spanned by $F(n)=\delta_{n 1}\left(\begin{array}{l}0 \\ 1\end{array}\right)$. Thus

$$
\ell_{2}^{H}(\{1, \ldots, N\}) \ominus Z=\ell_{2}^{H}(\{2, \ldots, N\}) \cong \ell_{2}(\{2, \ldots, N\}),
$$

as expected.

\section{REFERENCES}

[1] D.Z. Arov and H. Dym, J-inner matrix functions, interpolation and inverse problems for canonical systems, I: Foundations, Int. Eq. Op. Theory 29 (1997), 373-454. MR1484860 (99c:47011)

[2] F.V. Atkinson, Discrete and Continuous Boundary Problems, Academic Press, New York, 1964. MR0176141(31:416)

[3] E.A. Coddington and N. Levinson, Theory of Ordinary Differential Equations, McGraw-Hill, New York, 1972. MR0069338 (16:1022b)

[4] L. de Branges, Hilbert Spaces of Entire Functions, Prentice-Hall, Englewood Cliffs, 1968. MR0229011 (37:4590)

[5] W.F. Donoghue, Monotone Matrix Functions and Analytic Continuation, Springer-Verlag, Berlin, 1974. MR0486556 (58:6279)

[6] W.N. Everitt and L. Markus, Multi-interval linear ordinary boundary value problems and complex symplectic algebra, Memoirs Amer. Math. Soc. 715 (2001). MR 1828557 (2002i:34154)

[7] F. Gesztesy and E. Tsekanovskii, On matrix-valued Herglotz functions, Math. Nachr. 218 (2000), 61-138. MR.1784638 (2001j:47018)

[8] S. Hassi, H. de Snoo, and H. Winkler, Boundary-value problems for two-dimensional canonical systems, Int. Eq. Op. Theory 36 (2000), 445-479. MR1759823 (2002e:47054)

[9] D. Hinton and A. Schneider, On the Titchmarsh-Weyl coefficients for singular $S$-Hermitian systems I, Math. Nachr. 163 (1993), 323-342. MR1235076 (94j:34027)

[10] Y. Last and B. Simon, Eigenfunctions, transfer matrices, and absolutely continuous spectrum of one-dimensional Schrödinger operators, Invent. Math. 135 (1999), 329-367. MR:1666767 (2000f:47060)

[11] V.F. Lazutkin, KAM Theory and Semiclassical Approximations to Eigenfunctions, SpringerVerlag, Berlin, 1993. MR1239173 (94m:58069)

[12] D. McDuff and D. Salamon, Introduction to Symplectic Topology, Clarendon Press, Oxford, 1995. MR1373431 (97b:58062)

[13] J.W. Milnor and J.D. Stasheff, Characteristic Classes, Princeton University Press, Princeton, 1974. MR0440554 (55:13428)

[14] S.A. Orlov, Nested matrix discs analytically depending on a parameter and theorems on the invariance of ranks of radii of limiting discs, Math. USSR Izv. 10 (1976), 565-613. 
[15] C. Remling, Schrödinger operators and de Branges spaces, J. Funct. Anal. 196 (2002), 323394. MR.1943095 (2003j:47055)

[16] L.A. Sakhnovich, Spectral Theory of Canonical Systems: Method of Operator Identities, Birkhäuser, Basel, 1999. MR1690379 (2000e:47073)

Fachbereich Mathematik, Universität Osnabrück, 49069 Osnabrück, Germany

Department of Mathematics, University of Oklahoma, Norman, Oklahoma 73019

E-mail address: cremling@math.ou.edu

$U R L$ : www.math.ou.edu/ cremling 